\title{
Undernutrition and associated factors in primary schoolchildren in Lokossa, Benin: a cross-sectional study
}

\author{
Desnutrição e fatores associados em escolares de escolas primária em Lokossa, Benin: um estudo transversal
}

Desnutrición y factores asociados en estudiantes de primaria en Lokossa, Benin: un estudio transversal

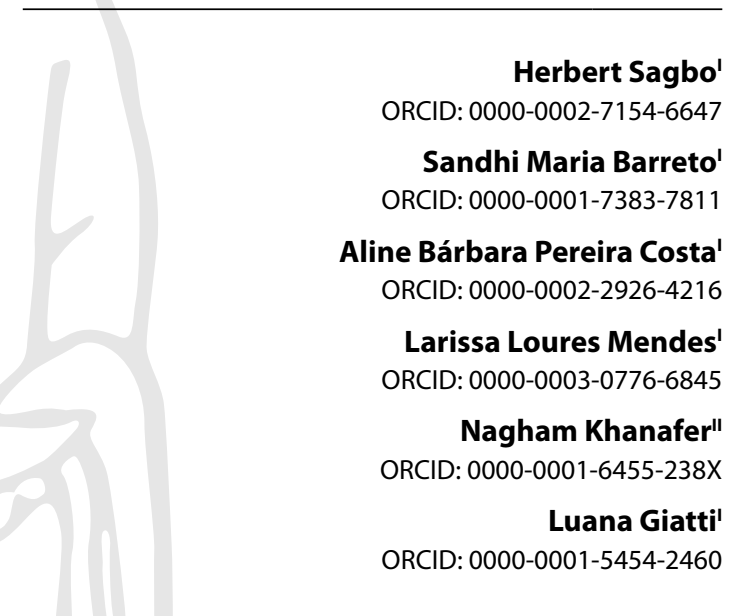

'Universidade Federal de Minas Gerais. Belo Horizonte, Minas Gerais, Brazil. "Université Claude Bernard Lyon 1. Lyon, Rhône-Alpes, France.

How to cite this article: Sagbo H, Barreto SM, Costa ABP, Mendes LL, Khanafer N, Giatti L. Undernutrition and associated factors in primary schoolchildren in Lokossa, Benin: a cross-sectional study. Rev Bras Enferm. 2022;75(3):e20210254. https://doi.org/10.1590/0034-7167-2021-0254

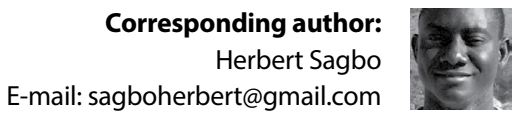

EDITOR IN CHIEF: Dulce Barbosa ASSOCIATE EDITOR: Carina Dessotte

Submission: 04-02-2021

Approval: 09-01-2021

\begin{abstract}
Objectives: to estimate the prevalence of stunting and thinness and sociodemographic, household, family, dietary, and scholar associated factors in schoolchildren living in LokossaBenin. Methods: a survey conducted in a probabilistic sample $(n=615)$ of primary schoolchildren (8-17 years), from 12/2018 to 01/2019, using structured questionnaire and 24-hour dietary recall. Thinness and stunting were defined as Height-for-age and Body Mass Index-for-age below -2 standard deviations, respectively. Associations were estimated by chi-square test and logistic regression. Results: thinness and stunting prevalence was 13.1\% (95\% Cl:9.0$18.7)$ and $25.5 \%(95 \% \mathrm{Cl}: 20.6-31.2)$, respectively. Odds of thinness were higher among older schoolchildren and those who experienced hunger at school. Odds of stunting increased with age, low diet diversity, experiencing hunger at school, and having school meal five days a week (OR:2.09; $95 \% \mathrm{Cl}: 1.29-3.36)$. Conclusions: stunting was the most common problem. Older schoolchildren and those with food deprivation or poor diet diversity were the most affected. Descriptors: Malnutrition; Risk Factors; Child; Adolescent; Benin.
\end{abstract}

\section{RESUMO}

Objetivos: estimar a prevalência de baixa estatura e magreza em escolares de Lokossa-Benin e fatores sociodemográficos, domiciliares, familiares, alimentares e da escola associados. Métodos: inquérito em amostra probabilística $(n=615)$ de escolares com idade entre 8 e 17 anos usando questionário estruturado e recordatório-24 horas. Baixa estatura e magreza foram definidas como Estatura-para-idade e Índice de Massa Corporal-para-idade inferior a -2 desvios-padrão, respectivamente. Associações foram estimadas pelo Teste do Qui-Quadrado e regressão logística. Resultados: prevalências de magreza e baixa estatura foram 13,1\% (IC95\%: 9,0-18,7) e 25,5\% (IC95\%: 20,6-31,2). As chances de magreza foram maiores entre escolares mais velhos e que relataram sentir fome na escola. Chances de baixa estatura aumentaram com idade, baixa diversidade alimentar, relatar fome na escola e merenda escolar cinco dias/semana (OR:2,09; IC95\%:1,29-3,36). Conclusões: a baixa estatura foi o problema mais comum e escolares mais velhos, com privação alimentar ou baixa diversidade alimentar foram os mais afetados.

Descritores: Desnutrição; Fatores de Risco; Criança; Adolescente; Benin.

\section{RESUMEN}

Objetivos: estimar la prevalencia de baja estatura y delgadez en estudiantes de LokossaBenin y factores sociodemográficos, domésticos, familiares, dietéticos y escolares asociados. Métodos: encuesta en una muestra probabilística $(n=615)$ de escolares de 8 a 17 años mediante cuestionario estructurado y recordatorio de 24 horas. La baja estatura y la delgadez se definieron como la talla para la edad y el índice de masa corporal para la edad inferior a -2 desviaciones estándar, respectivamente. Las asociaciones se estimaron mediante la prueba de chi-cuadrado y regresión logística. Resultados: las prevalencias de delgadez y baja estatura fueron 13,1\% (IC95\%:9,0-18,7) y 25,5\% (IC95\%:20,6-31,2). Las probabilidades de estar delgadas eran más altas entre los estudiantes mayores que informaron tener hambre en la escuela. Las posibilidades de estatura baja aumentaron con la edad, la diversidad dietética baja, y los informes sobre el hambre en la escuela y los almuerzos escolares cinco días a la semana (OR:2,09; IC95\%:1,29-3,36). Conclusiones: la baja estatura fue el problema más común y los estudiantes mayores, con privación de alimentos o baja diversidad alimentaria fueron los más afectados.

Descriptores: Desnutrición; Factores de Riesgo; Niño; Adolescente; Benin. 


\section{INTRODUCTION}

In spite of growing obesity-related concerns, undernutrition remains a public health concern and has been rising in the world since $2015^{(1)}$. Undernutrition manifests as wasting (acute malnutrition), stunting (chronic malnutrition), underweight, and mineral and vitamin deficiencies or excessiveness ${ }^{(2)}$. In 2017, the global prevalence of stunting and underweight in children aged under 5 years was $22 \%$ and $7.5 \%$, respectively ${ }^{(3)}$. Malnutrition is of particular relevance in Africa, where an estimated 256.1 million children, $90 \%$ of whom lived in sub-Saharan Africa, were undernourished in 2018, with a prevalence of $30 \%$ and $7.1 \%$, respectively, for stunting and underweight among children aged up to 5 years ${ }^{(1)}$.

Expected weight gain and growth in childhood and adolescence are largely dependent on appropriate quality and quantity of foods, and are highly impacted by environmental and socioeconomic factors ${ }^{(4)}$, such as maternal level of education, household income, sanitary conditions, and access to and use of healthcare services ${ }^{(5)}$. Political, economic, social and cultural settings restricting access to essential resources and human rights are basic causes of malnutrition and may persist across generations, giving rise to a vicious cycle(6).

Lack of energy and nutrients required for proper growth in childhood has potentially lifelong negative effects on health, physical growth and intellectual development ${ }^{(7)}$. Nutritional deficiency is one of the major risk factors for death in the first years of life ${ }^{(8)}$. Persistent malnutrition throughout school age and adolescence enhances somatic growth deficit, delays sexual maturity, and hinders development of cognitive and emotional skills acquired in this phase of life $\mathrm{e}^{(9)}$.

School age is a vital period for physical growth and mental development and also a stage of life with high health vulnerability ${ }^{(5)}$. This stage of life may also represent a window of opportunity for at least partially offsetting the effects of malnutrition at a younger age. Studies investigating individual, household- and school-related factors may provide relevant information to support more effective public policies to address this significant public health concern.

School environments provide an opportunity for timely interventions to prevent a range of conditions, including undernutrition. Schools are increasingly becoming key settings for health promoting actions directed to children and adolescents. Globally, most children and adolescents are enrolled in school even in the most remote rural areas of low-income countries. For these reasons, school health programs have become leading strategies to safeguard schoolchildren's health and well-being. For instance, the initiative "Making Every School a Health Promoting School", from the World Health Organization (WHO) and United Nations Educational, Scientific and Cultural Organization (UNESCO), was launched to assist over to 2,3 billion schoolchildren until $2023^{(10)}$.

In Benin, in a sub-Saharan African country with a population of approximately 10 million people, an estimated $9.6 \%$ of the population experienced food insecurity in $2017^{(11)}$, and the prevalence of stunting, underweight, and wasting in children under five years of age reached $32 \%, 17 \%$ and $5 \%$, respectively, in $2018^{(12)}$. This situation appears even worse in rural areas of Benin, where a study with schoolchildren aged 5-10 years, in 2015, reported a prevalence of underweight, wasting, and stunting as $40 \%, 31 \%$ and $22 \%$, respectively ${ }^{(13)}$.

\section{OBJECTIVES}

To estimate the prevalence of stunting and thinness, and also to investigate sociodemographic, household- and family-related, dietary, and scholar factors associated to these conditions in primary schoolchildren living in Lokossa-Benin.

\section{METHODS}

\section{Ethics approval and consent to participate}

Participants and parents (or guardians) signed an Informed Assent Form and an Informed Consent Form, respectively, for inclusion before they participated in the study. The protocol was approved by the National Health Research Ethics Committee from Benin, Opinion 28 of September 20, 2018.

\section{Study population}

A cross-sectional study based on data extracted from the Research on Nutritional and Health Status of Primary Education Students in the city of Lokossa-Benin. Data were collected from a representative sample of sixth-year primary schoolchildren, attending public and private schools located in rural and urban areas of Lokossa, between December/2018 and January/2019. This municipality is located in the Mono Department, in the southern region of the country, where infrastructure and services are largely concentrated. According to the 2013 population census, $45 \%$ of Lokossa population was aged less than 15 years ${ }^{(14)}$. This sample comprised exclusively schoolchildren in the last year of primary school, since those in previous years would not be able to properly answer the questionnaire used for data collection.

Schoolchildren in this sample were selected using conglomerate probabilistic sampling with two strata (urban and rural). Schools (conglomerates) with probability of selection proportional to the number of schoolchildren were chosen in each stratum. All sixth-year schoolchildren attending the selected schools on the day of data collection were invited to participate.

Sampling selection was based on the list of schools and number of schoolchildren enrolled in the sixth year of the previous school year (2017/2018), provided by Mono Education Department. Outcomes of interest were estimated with a level of significance of $5 \%$, prevalence of condition of interest of $10 \%$, and maximum error of $3.25 \%$. A design effect of 1.25 was also accounted for based on similar previous investigations ${ }^{(15)}$. A calculation method accounting for maximum possible sample size (Sample Size for Finite Populations, fcp) was also adopted. Based on these parameters, the required sample size was 667 individuals. Only 660 schoolchildren attended the 26 selected schools that school year. Of these, 35 refused to participate and 10 were absent on the day of data collection. The final sample comprised 615 schoolchildren aged 8 to 17 years.

\section{Data collection}

The following instruments were used to build the structured questionnaire adopted in the present survey: 1) Global SchoolBased Student Health Survey (GSHS-Benin 2009), based on the 
WHO standard GSHS instrument ${ }^{(16)}$; 2) Brazilian National Adolescent School-based Health Survey (PeNSE-2015), of the Brazilian Ministry of Health ${ }^{(17)}$; 3) Study on Cardiovascular Risks in Adolescents (ERICA), carried out in Brazil ${ }^{(18)}$; and 4) the questionnaire used to assess the double burden of malnutrition during school age in urban areas of Burkina Faso-2008-2009(19).

Firstly, the above instruments were assessed, and the questions were selected and revised contemplating common lexis and culture of Benin schoolchildren. The order of the questions and the instructions on how to respond them was also revised considering the education level of the study sample. Then, the questionnaire was translated into French by a native researcher (HS) and double checked by a French research (NK). The final version of the questionnaire comprised the following thematic areas: a) sociodemographic characteristics, household and school settings, dietary behaviours, alcohol use, physical activity, protective factors, mental health, hygiene and oral health, and an overall appreciation of the questionnaire.

The final version of the questionnaires and instruments were pretested using two school classes with 26 and 27 schoolchildren each in Lokossa-Benin. After pretest, the questionnaire was shortened and some questions adjusted, to improve their comprehension. Finally, two pilot studies were carried out in three differents school classes to test and refine all data collection instruments and procedures. The school classes participating in pretest or pilot studies were not included in the study sample.

After having completed the questionnaire in the classroom, schoolchildren were sent for anthropometric measurements. Body weight was measured using a portable electronic scale with $150 \mathrm{~kg}$ capacity and $0.1 \mathrm{~kg}$ accuracy (Seca 803, Hamburg), with schoolchildren wearing light clothes and barefoot. Height was measured using a mobile stadiometer with millimetre resolution (Seca 213, Hamburg), while schoolchildren stood on a platform with the back of the heels and occipital bone pressed against the stadiometer and looking straight ahead.

Schoolchildren completed a 24-hour dietary recall the day after questionnaire completion. The multi-pass method ${ }^{(20)}$ was used to minimize memory bias and food intake underreporting.

Data was collected by trained and certified undergraduate nutritional science students. Two pilot studies were performed to test and refine the data collection instruments and procedures. Filled out research questionnaires and forms were typed with double-checking.

\section{Nutritional status}

Body Mass Index (BMI) was calculated using body weight in kilograms divided by the square of height in meters. Heightfor-age (HAZ) and BMI-for-age (BAZ) indices were calculated, standardized according to Z-scores and categorized according to WHO cut-offs for children and adolescents aged 5 to 19 years ${ }^{(21)}$. Indicators were estimated using the software WHO AnthroPlus (World Health Organization, Switzerland). Thinness and stunting were defined as $\mathrm{BAZ}<-2 \mathrm{Z}$-score and $\mathrm{HAZ}<-2 \mathrm{Z}$-score, respectively.

\section{Explanatory variables}

Sociodemographic characteristics included gender and age in years, grouped into age categories $(8-10 ; 11-12 ; 13-14 ; \geq 15)$.
Household and family context comprised maternal and paternal education (noneducated, primary education, secondary education, university, and no information), parental work status (employed, unemployed), and household socioeconomic status. Household socioeconomic status was estimated by adding the following household characteristics and goods: type of construction, number of bedrooms, type of bed, access to treated water, access to electricity, type of toilet, household car availability, and monthly allowance. Adding up-values yielded a score ranging from 8 to 21, categorized into tertiles (high, medium and low) ${ }^{(22)}$. Other variables included were living with parents (both, mother only, father only, others), family size (i.e., number of household members) categorized into up to 7 or $\geq 8$, and household location (urban or rural).

Dietary characteristics were food diversity score, weekly breakfast frequency ( $>5$ days, $<5$ days per week) and experiencing hunger at home and at school (never, rarely, sometimes, many times/ always). The dietary diversity score was estimated from 24-hour dietary recall data. Foods identified in recalls were categorized into 16 groups following the recommendations of FAO/FANTA Guidelines: cereals, milk and dairy products, eggs, fats, vitamin A-rich fruits and vegetables, fish, other fruits and vegetables, dark green vegetables, and meats. For score calculation purposes, "1" and " 0 " were attributed to presence or absence of a given food group in the diet. Therefore, values attributed to each of the nine food groups were summed. Scores were categorized into three dietary diversity levels: low ( $\leq 3$ food groups), medium (4-5 food groups) and high ( $\geq 6$ food groups) ${ }^{(23)}$.

School-related characteristics were type of school (public or private) and school meal availability ( $<5$ days a week, 5 days a week).

\section{Statistical analysis}

Venn diagram was used to describe the nutritional status based on thinness and stunting. Study population characteristics were described according to thinness and stunting using proportions. The prevalence of thinness and stunting with $95 \%$ confidence intervals $(95 \% \mathrm{Cl})$ were estimated according to explanatory variables. Prevalence differences according to explanatory variables were estimated using Pearson's chi-square test, with a significance level of $<0.05$.

The magnitude of associations between explanatory variables and nutritional status was estimated according to Odds Ratio (OR) and respective $95 \% \mathrm{Cl}$ obtained using logistic regression models. Crude models were obtained and adjusted for gender, with a significance level of $<0.05$. We used the Generalized Estimating Equation model that considers the correlation among schoolchildren within the schools and models the average population response as a function of covariables ${ }^{(24)}$.

Analyses were conducted using the software Stata 14.0 (Stata Corporation, College Station, United States). Stata survey commands corrected for sampling weight, design effect and finite sample size were used.

\section{RESULTS}

Three out of the 615 selected schoolchildren were excluded from the analysis due to lack of nutritional status data, and the final sample comprised 612 students. Age ranged from 8 to 17 
years, with $72.2 \%$ aged over 10 years. Most schoolchildren were males (55.6\%), lived in urban areas (61.9\%) and attended public schools (69.8\%) (Table 1).

The overall prevalence of thinness and stunting among primary schoolchildren of Lokossa were $13.1 \%$ (95\%Cl: 9.0-18.7) and $25.5 \%$ (95\%Cl: 20.6-31.2), respectively. Figure 1 displays the percentage of schoolchildren presenting only stunting (19.3\%), only thinness (6.8\%), and both conditions simultaneously (6.3\%). Only $67.6 \%$ of the schoolchildren had adequate nutritional status.

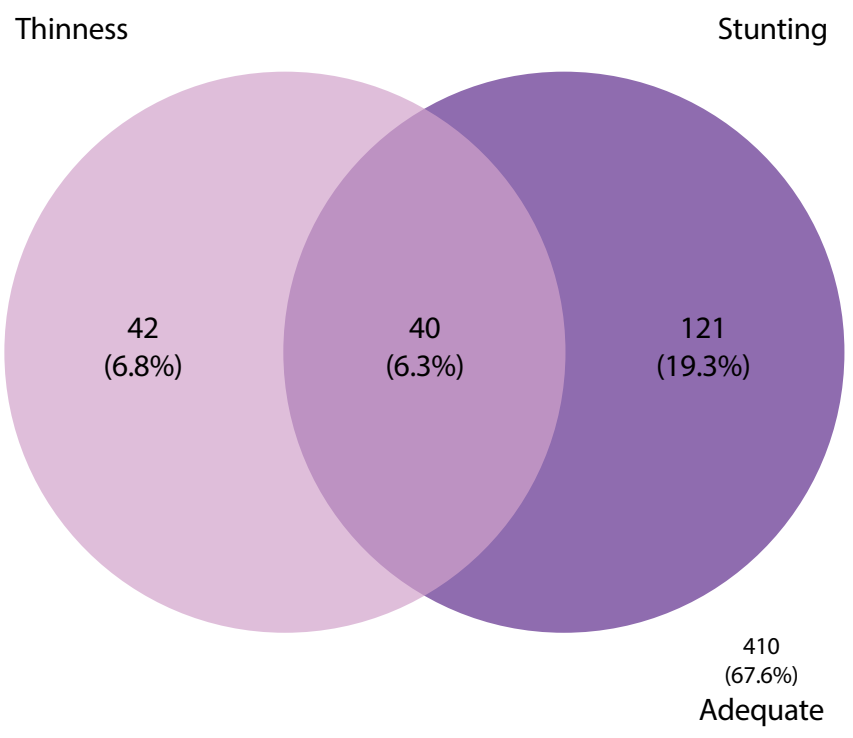

The percentages presented consider the sample weight.

Figure 1 - Venn diagram of the frequency of the nutritional status of primary schoolchildren, Lokossa, Benin, December 2018 / January 2019

There was no statistically significant differences on the prevalence of thinness according to schoolchildren' sociodemographic characteristics and household and family context. However, the prevalence of thinness was higher in schoolchildren experiencing hunger at school many times/always $(p=0.015)$ and receiving school meals five days a week $(p=0.049)$. The prevalence of stunting increased with age and was higher among boys $(p=0.001)$, offspring of unemployed mothers $(p=0.029)$, unemployed fathers $(p=0.020)$, schoolchildren living in a household of low socioeconomic status $(p=0.037)$, rural areas $(p=0.031)$, experiencing hunger at home $(p=0.030)$, and receiving school meals 5 days a week $(p=0.01)$ (Table 1).

Results of logistic regression analysis of associations between explanatory variables and nutritional status are shown in Table 2. After adjustment for gender, the age range of 13-14 and >15 presented more than three times the odds of thinness (OR:3.54; 95\%Cl1.57-8.03 and OR:3.23 95\%Cl:1.08-9.61, respectively) when compared to 8-10-years. Besides, experiencing hunger at

Results of logistic regression analysis of associations between explanatory variables and nutritional status are shown in Table 2. After adjustment for gender, the age range of 13-14 and $>15$ presented more than three times the odds of thinness (OR:3.54; 95\%Cl1.57-8.03 and OR:3.23 95\%Cl:1.08-9.61, respectively) when compared to 8-10-years. Besides, experiencing hunger at many times/always versus never experiencing hunger at school was also associated with more than two times the odds of thinness (OR:2.68; 95\%Cl:1.19-6.04), regardless of gender.

Analysis adjusted for gender showed that odds of stunting strongly increased with age. These analyses also displayed that odds of stunting was $79 \%$ higher in presence of low diversity of diet (95\%Cl:1.07-2.98) versus high diversity of diet, $168 \%$ (95\%Cl:1.19-6.04) higher among those reported experiencing hunger at school many times/always versus never experiencing, and $109 \%$ (95\% Cl: 1.29-3.36) higher among those reported eating school meal $>5$ days a week versus $<5$ days a week. University maternal education (vs non-educated) decreased odds of stunting (OR:0.36; 95\%Cl:0.18-0.70) after adjustment for gender (Table 2).

Table 1 - Distribution of the study population and the prevalence of thinness and stunting according to sociodemographic characteristics, household and family context, and school-related characteristics in primary schoolchildren, Lokossa, Benin, December 2018 / January 2019

\begin{tabular}{|c|c|c|c|c|c|}
\hline \multirow[b]{2}{*}{ Variables } & \multirow[b]{2}{*}{$\begin{array}{c}\text { Total } \\
(n=612) \\
\%\end{array}$} & \multicolumn{4}{|c|}{ Prevalence } \\
\hline & & $\begin{array}{l}\text { Thinness } \\
(n=612) \\
\%(95 \% \mathrm{Cl})\end{array}$ & $p$ value & $\begin{array}{c}\text { Stunting } \\
(\mathbf{n}=612) \\
\%(95 \% \mathrm{Cl})\end{array}$ & $p$ value \\
\hline \multicolumn{6}{|c|}{ Sociodemographic characteristics } \\
\hline $\begin{array}{l}\text { Sex } \\
\quad \text { Male } \\
\text { Female }\end{array}$ & $\begin{array}{l}55.6 \\
44.4\end{array}$ & $\begin{array}{l}12.9(8.4-19.3) \\
13.4(8.7-20.0)\end{array}$ & 0.845 & $\begin{array}{l}31.3(24.9-38.5) \\
18.3(13.0-25.0)\end{array}$ & 0.001 \\
\hline $\begin{array}{l}\text { Age } \\
\text { 8-10 years } \\
11-12 \text { years } \\
13-14 \text { years } \\
\geq 15 \text { years }\end{array}$ & $\begin{array}{c}27.7 \\
42.7 \\
24.8 \\
4.8\end{array}$ & $\begin{array}{c}9.7(4.0-22.0) \\
10.0(5.0-18.9) \\
20.7(15.7-26.9) \\
20.8(12.0-33.5)\end{array}$ & 0.06 & $\begin{array}{c}6.7(3.4-12.7) \\
31.0(25.0-37.7) \\
35.5(26.3-45.8) \\
34.0(23.5-46.5)\end{array}$ & 0.001 \\
\hline \multicolumn{6}{|c|}{ Household and family context } \\
\hline $\begin{array}{l}\text { Maternal education } \\
\text { Noneducated } \\
\text { Primary } \\
\text { Secondary } \\
\text { University } \\
\text { No information }\end{array}$ & $\begin{array}{c}42.2 \\
28.2 \\
17.6 \\
3.9 \\
8.1\end{array}$ & $\begin{array}{c}16.2(11.7-21.9) \\
11.7(6.2-21.0) \\
9.3(4.6-17.7) \\
10.5(5.7-18.4) \\
11.3(5.9-20.6)\end{array}$ & 0.182 & $\begin{array}{c}26.7(2.9-33.4) \\
28.1(19.3-38.9) \\
22.6(16.0-30.9) \\
10.5(5.7-18.4) \\
24.0(14.2-37.6)\end{array}$ & 0.261 \\
\hline
\end{tabular}




\begin{tabular}{|c|c|c|c|c|c|}
\hline \multirow[b]{2}{*}{ Variables } & \multirow[b]{2}{*}{$\begin{array}{c}\begin{array}{c}\text { Total } \\
(\mathrm{n}=612) \\
\%\end{array} \\
\end{array}$} & \multicolumn{4}{|c|}{ Prevalence } \\
\hline & & $\begin{array}{c}\text { Thinness } \\
(n=612) \\
\%(95 \% \mathrm{Cl})\end{array}$ & $p$ value & $\begin{array}{c}\text { Stunting } \\
(n=612) \\
\%(95 \% \mathrm{Cl})\end{array}$ & $p$ value \\
\hline Paternal education & & & 0.398 & & 0.188 \\
\hline Noneducated & 12.7 & $10.4(5.2-19.7)$ & & $29.6(20.4-40.7)$ & \\
\hline Primary & 26.2 & $17.3(11.1-26.1)$ & & $28.9(21.3-37.8)$ & \\
\hline Secondary & 36.9 & $11.9(7.3-18.7)$ & & $26.6(20.1-34.3)$ & \\
\hline University & 16.8 & $10.9(5.0-22.1)$ & & $14.5(7.2-26.7)$ & \\
\hline No information & 7.4 & $10.1(4.6-20.6)$ & & $23.4(10.8-43.7)$ & \\
\hline Mother's occupation $(n=606)$ & & & 0.469 & & 0.029 \\
\hline Employed & 90.9 & $12.8(8.8-18.2)$ & & $24.0(19.6-29.1)$ & \\
\hline Unemployed & 9.1 & $16.6(7.1-34.0)$ & & $40.5(25.2-57.9)$ & \\
\hline Father's occupation $(\mathrm{n}=592)$ & & & 0.384 & & 0.020 \\
\hline Employed & 99.5 & $12.9(8.9-18.5)$ & & $25.5(20.3-31.6)$ & \\
\hline Unemployed & 0.5 & $24.2(5.2-64.9)$ & & $78.8(24.0-97.7)$ & \\
\hline Household socioeconomic status ( $n=590$ ) & & & 0.191 & & 0.037 \\
\hline High & 38.9 & $10.9(7.6-15.5)$ & & $20.4(14.7-27.6)$ & \\
\hline Medium & 24.9 & $10.4(3.8-25.4)$ & & $22.5(16.2-30.4)$ & \\
\hline Low & 36.2 & $17.2(11.8-24.2)$ & & $31.9(23.9-41.2)$ & \\
\hline Living with parents ( $n=606)$ & & & 0.397 & & 0.634 \\
\hline Both & 74.3 & $11.9(7.3-18.9)$ & & $24.5(19.1-30.9)$ & \\
\hline Mother only & 10.4 & $17.2(8.5-31.6)$ & & $31.7(19.8-46.7)$ & \\
\hline Father only & 5.2 & $20.3(11.0-34.3)$ & & $23.7(12.5-40.5)$ & \\
\hline Others & 10.1 & $13.5(7.7-22.5)$ & & $10.2(15.3-37.5)$ & \\
\hline Family size $(n=609)$ & & & 0.622 & & 0.949 \\
\hline $2-7$ & 58.1 & $12.2(6.5-21.8)$ & & $25.3(18.9-32.9)$ & \\
\hline$\geq 8$ & 41.9 & $14.2(10.7-18.5)$ & & $25.6(19.8-32.3)$ & \\
\hline Household location & & & 0.968 & & 0.031 \\
\hline Urban & 61.9 & $13.2(7.4-22.3)$ & & $20.8(14.6-28.7)$ & \\
\hline Rural & 38.1 & $12.9(9.2-17.9)$ & & $33.2(25.1-42.4)$ & \\
\hline Dietary diversity $(n=603)$ & & & 0.113 & & 0.531 \\
\hline High & 41.7 & $15.8(9.5-25.1)$ & & $24.2(18.1-31.5)$ & \\
\hline Medium & 34.4 & $8.9(5.3-14.6)$ & & $26.4(20.1-33.8)$ & \\
\hline Low & 23.9 & $20.4(11.6-33.3)$ & & $31.2(18.4-47.5)$ & \\
\hline Breakfast $(n=610)$ & & & 0.288 & & 0.453 \\
\hline$>5$ days & 42.4 & $15.5(8.2-27.5)$ & & $23.2(15.8-32.6)$ & \\
\hline$<5$ days & 57.6 & $11.2(8.1-15.2)$ & & $27.2(21.1-34.3)$ & \\
\hline Experiencing hunger at home & & & 0.727 & & 0.030 \\
\hline Never & 64.9 & $12.1(7.2-19.6)$ & & $24.6(19.3-30.1)$ & \\
\hline Rarely & 18.7 & $13.5(8.9-20.1)$ & & $25.0(16.3-36.4)$ & \\
\hline Sometimes & 11.2 & $16.7(8.6-29.7)$ & & $27.9(9.6-58.4)$ & \\
\hline Many times/always & 5.2 & $15.6(6.5-32.8)$ & & $33.9(18.9-53.1)$ & \\
\hline Experiencing hunger at school & & & 0.015 & & 0.075 \\
\hline Never & 65.7 & $12.3(8.4-17.7)$ & & $22.3(18.2-26.9)$ & \\
\hline Rarely & 15.3 & $15.7(8.2-27.9)$ & & $30.6(18.5-46.2)$ & \\
\hline Sometimes & 13.1 & $6.6(2.6-16.1)$ & & $26.5(18.4-36.5)$ & \\
\hline Many times/always & 5.9 & $28.7(17.4-43.6)$ & & $46.2(23.1-71.0)$ & \\
\hline School-related characteristics & & & & & \\
\hline School type & & & 0.462 & & 0.450 \\
\hline Public & 69.8 & $11.9(8.5-16.5)$ & & $26.9(20.3-34.7)$ & \\
\hline Private & 30.2 & $15.8(7.6-28.9)$ & & $22.3(14.1-33.5)$ & \\
\hline School meal & & & 0.049 & & 0.001 \\
\hline$<5$ days a week & 93.6 & $12.5(8.6-17.8)$ & & $24.0(18.9-29.9)$ & \\
\hline 5 days a week & 6.4 & $13.2(9.1-18.9)$ & & $25.4(20.3-31.2)$ & \\
\hline
\end{tabular}

The percentages presented consider the sample weight.

Table 2 - Odds Ratio and $95 \%$ confidential intervals of sociodemographic characteristics, household and family context, and school-related characteristics with thinness and stunting in primary schoolchildren, Lokossa, Benin, December 2018 / January 2019

\begin{tabular}{|c|c|c|c|c|}
\hline \multirow[b]{2}{*}{ Variables } & \multicolumn{2}{|c|}{ Thinness } & \multicolumn{2}{|c|}{ Stunting } \\
\hline & $\begin{array}{c}\text { Crude } \\
\text { OR }(95 \% \mathrm{Cl})\end{array}$ & $\begin{array}{l}\text { Adjusted } \\
\text { OR (95\%Cl) }\end{array}$ & $\begin{array}{c}\text { Crude } \\
\text { OR }(95 \% \mathrm{Cl})\end{array}$ & $\begin{array}{l}\text { Adjusted } \\
\text { OR (95\%Cl) }\end{array}$ \\
\hline \multicolumn{5}{|l|}{ Sex } \\
\hline Male & 1.00 & 1.00 & 1.00 & 1.00 \\
\hline Female & $0.97(0.59-1.57)$ & $0.71(0.43-1.15)$ & $0.52(0.36-0.74)^{*}$ & $0.72(0.44-1.19)$ \\
\hline \multicolumn{5}{|l|}{ Age } \\
\hline 8-10 years & 1.00 & 1.00 & 1.00 & 1.00 \\
\hline $11-12$ years & $1.22(0.57-2.63)$ & $1.23(0.57-2.64)$ & $5.27(2.75-10.09)^{* * *}$ & $5.00(2.64-9.48)^{* *}$ \\
\hline $13-14$ years & $3.53(1.57-7.94)^{*}$ & $3.54(1.57-8.03)^{*}$ & $6.42(3.09-13.32)^{* * *}$ & $6.09(2.97-12.48)^{* *}$ \\
\hline$\geq 15$ years & $3.22(1.18-9.29)^{*}$ & $3.23(1.08-9.61)^{*}$ & $7.25(3.84-13.68)^{* * *}$ & $6.75(3.69-12.37)^{* *}$ \\
\hline
\end{tabular}




\begin{tabular}{|c|c|c|c|c|}
\hline \multirow[b]{2}{*}{ Variables } & \multicolumn{2}{|c|}{ Thinness } & \multicolumn{2}{|c|}{ Stunting } \\
\hline & $\begin{array}{c}\text { Crude } \\
\text { OR }(95 \% \mathrm{CI})\end{array}$ & $\begin{array}{c}\text { Adjusted } \\
\text { OR }(95 \% \mathrm{CI})\end{array}$ & $\begin{array}{c}\text { Crude } \\
\text { OR }(95 \% \mathrm{CI})\end{array}$ & $\begin{array}{c}\text { Adjusted } \\
\text { OR }(95 \% \mathrm{CI})\end{array}$ \\
\hline \multicolumn{5}{|l|}{ Maternal education } \\
\hline Noneducated & 1.00 & 1.00 & 1.00 & 1.00 \\
\hline Primary & $0.79(0.51-1.22)$ & $0.79(0.51-1.22)$ & $1.13(0.81-1.57)$ & $1.14(0.82-1.58)$ \\
\hline Secondary & $0.80(0.33-1.93)$ & $0.80(0.33-1.92)$ & $1.05(0.68-1.62)$ & $1.07(0.70-1.64)$ \\
\hline University & $0.64(0.30-1.35)$ & $0.64(0.30-1.35)$ & $0.37(0.20-0.67)$ & $0.36(0.18-0.70)^{*}$ \\
\hline No information & $0.92(0.52-1.62)$ & $0.97(0.59-1.59)$ & $0.86(0.46-1.61)$ & $0.94(0.48-1.81)$ \\
\hline \multicolumn{5}{|l|}{ Paternal education } \\
\hline Noneducated & 1.00 & 1.00 & 1.00 & 1.00 \\
\hline Primary & $1.38(0.58-3.29)$ & $1.38(0.58-3.29)$ & $1.08(0.65-1.76)$ & $1.08(0.65-1.76)$ \\
\hline Secondary & $1.10(0.51-2.35)$ & $1.10(0.51-2.37)$ & $1.11(0.70-1.72)$ & $1.11(0.70-1.72)$ \\
\hline University & $1.01(0.38-2.67)$ & $1.02(0.39-2.65)$ & $0.60(0.29-1.24)$ & $0.60(0.29-1.24)$ \\
\hline No information & $1.05(0.39-2.78)$ & $1.05(0.38-2.88)$ & $0.84(0.38-1.83)$ & $0.84(0.38-1.83)$ \\
\hline \multicolumn{5}{|l|}{ Mother's occupation } \\
\hline Employed & 1.00 & 1.00 & 1.00 & 1.00 \\
\hline Unemployed & $1.20(0.50-2.89)$ & $1.20(0.50-2.90)$ & $1.73(0.86-3.50)$ & $1.68(0.82-3.45)$ \\
\hline \multicolumn{5}{|l|}{ Father's occupation } \\
\hline Employed & 1.00 & 1.00 & 1.00 & 1.00 \\
\hline Unemployed & $2.07(0.35-12.19)$ & $2.07(0.35-12.22)$ & $8.39(0.48-144.40)$ & $8.95(0.63-127.04)$ \\
\hline \multicolumn{5}{|c|}{ Household socioeconomic status } \\
\hline High & 1.00 & 1.00 & 1.00 & 1.00 \\
\hline Medium & $0.77(0.34-1.73)$ & $0.77(0.34-1.73)$ & $1.11(0.67-1.82)$ & $1.11(0.70-1.78)$ \\
\hline Low & $1.52(0.97-2.36)$ & $1.52(0.98-2.36)$ & $1.57(1.00-2.49)^{*}$ & $1.52(0.97-2.38)$ \\
\hline \multicolumn{5}{|l|}{ Living with parents } \\
\hline Both & 1.00 & 1.00 & 1.00 & 1.00 \\
\hline Mother only & $1.40(0.79-2.47)$ & $1.40(0.79-2.47)$ & $1.29(0.74-2.25)$ & $1.34(0.74-2.40)$ \\
\hline Father only & $2.05(0.90-4.63)$ & $2.05(0.91-4.61)$ & $0.89(0.44-1.80)$ & $0.86(0.41-1.79)$ \\
\hline Others & $0.88(0.36-2.13)$ & $0.98(0.60-1.59)$ & $0.88(0.45-1.71)$ & $0.83(0.42-1.62)$ \\
\hline \multicolumn{5}{|l|}{ Family size } \\
\hline $2-7$ & 1.00 & 1.00 & 1.00 & 1.00 \\
\hline$\geq 8$ & $1.29(0.81-2.06)$ & $1.29(0.81-2.06)$ & $0.92(0.68-1.25)$ & $0.95(0.70-1.30)$ \\
\hline \multicolumn{5}{|l|}{ Household location } \\
\hline Urban & 1.00 & 1.00 & 1.00 & \\
\hline Rural & $0.92(0.55-1.54)$ & $0.92(0.55-1.54)$ & $1.83(1.02-3.27)^{*}$ & $1.70(0.96-3.02)$ \\
\hline \multicolumn{5}{|l|}{ Dietary diversity } \\
\hline High & 1.00 & 1.00 & 1.00 & 1.00 \\
\hline Medium & $0.65(0.33-1.26)$ & $0.65(0.33-1.26)$ & $1.31(0.81-2.12)$ & $1.34(0.84-2.13)$ \\
\hline Low & $1.66(0.90-3.06)$ & $1.66(0.90-3.06)$ & $1.75(1.03-2.97)^{*}$ & $1.79(1.07-2.98)^{*}$ \\
\hline \multicolumn{5}{|l|}{ Breakfast } \\
\hline$>5$ days & 1.00 & 1.00 & 1.00 & 1.00 \\
\hline$<5$ days & $0.76(0.44-1.30)$ & $0.76(0.44-1.30)$ & $1.29(0.78-1.86)$ & $1.18(0.77-1.82)$ \\
\hline \multicolumn{5}{|c|}{ Experiencing hunger at home } \\
\hline Never & 1.00 & 1.00 & 1.00 & 1.00 \\
\hline Rarely & $1.21(0.63-2.29)$ & $1.20(0.62-2.32)$ & $0.90(0.52-1.54)$ & $0.85(0.48-1.49)$ \\
\hline Sometimes & $1.48(0.77-2.81)$ & $1.47(0.77-2.81)$ & $0.81(0.32-2.05)$ & $0.82(0.33-2.03)$ \\
\hline Many times/always & $0.83(0.28-2.46)$ & $0.83(0.28-2.47)$ & $1.44(0.70-2.95)$ & $1.39(0.63-3.06)$ \\
\hline \multicolumn{5}{|c|}{ Experiencing hunger at home } \\
\hline Never & 1.00 & 1.00 & 1.00 & 1.00 \\
\hline Rarely & $1.18(0.66-2.09)$ & $1.17(0.66-2.08)$ & $1.44(0.86-2.42)$ & $1.38(0.83-2.32)$ \\
\hline Sometimes & $0.55(0.24-1.28)$ & $0.55(0.23-1.31)$ & $1.34(0.93-1.91)$ & $1.27(0.89-1.80)$ \\
\hline Many times/always & $2.68(1.19-6.03)^{*}$ & $2.68(1.19-6.04)^{*}$ & $2.82(1.30-6.11)^{*}$ & $2.85(1.32-6.17)^{*}$ \\
\hline \multicolumn{5}{|l|}{ School type } \\
\hline Public & 1.00 & 1.00 & 1.00 & 1.00 \\
\hline Private & $1.13(0.52-2.43)$ & $1.14(0.53-2.44)$ & $0.51(0.23-1.09)$ & $0.58(0.28-1.16)$ \\
\hline \multicolumn{5}{|l|}{ School meal } \\
\hline$<5$ days a week & 1.00 & 1.00 & 1.00 & 1.00 \\
\hline 5 days a week & $1.30(0.62-2.71)$ & $1.30(0.62-2.73)$ & $2.30(1.49-3.57)^{*}$ & $2.09(1.29-3.36)^{*}$ \\
\hline
\end{tabular}

OR-Odds Ratio; $\mathrm{Cl}$ - Confidence Interval; Models adjusted for age and sex; ${ }^{*} p \leq 0.05 ;{ }^{* *} p \leq 0.001$.

\section{DISCUSSION}

This study, conducted at primary schools located in rural and urban areas of Lokossa-Benin, demonstrated stunting in more than one quarter of schoolchildren and thinness in over one tenth of children. After considering gender in the analysis, we found that odds of thinness, especially of stunting, increased with age. Stunting was also more frequent among schoolchildren facing adverse nutritional conditions, indicated by low dietary diversity, and those having school meal every weekday. Experiencing hunger at school was associated with more than 2- and 3-folds increases in odds of thinness and stunting, respectively. In contrast, the university maternal education was a protective factor for stunting. 
The prevalence of stunting varies widely among studies in the African continent, such as Guinea-Bissau (15.5\%) in $2011^{(25)}$, Ethiopia (5.2\%) in 2015(26) and Burkina-Faso (29.4\%) in 2015(27). Part of the differences observed in the prevalence of stunting can be attributed to sampling variations in the age groups included, such as 10-19 years in the study conducted in Ethiopia ${ }^{(25)}, 8-14$ years in the study from Burkina-Faso ${ }^{(27)}$, and 8-17 years in this study.

The frequency of thinness found in this study (13\%) was higher than that found in a southern Ethiopian study $(4.7 \%)^{(26)}$ and close to those reported in central Burkina Faso (11\%)(27) and in primary schoolchildren of Guinea Bissau (13\%) ${ }^{(25)}$. The variability in thinness prevalence among studies may be attributed to the inclusion or not of schoolchildren from rural areas, as it is generally greater in rural than urban areas. For instance, while the southern Ethiopian study ${ }^{(26)}$ included only urban schoolchildren, the Guinea Bissau study had only rural schoolchildren. It is important to note that the above quoted studies were conducted prior to 2015 and global malnutrition rates are rising since then ${ }^{(1)}$. Socioeconomic, environmental and cultural differences between study settings, plus variation in school attending rates among African countries may also explain prevalence variations in stunting and thinness among studies.

Stunting, the major problem identified in this study, stems from long-term food deprivation, especially micronutrients and protein inadequate intake, which could include prenatal nutrition and little maternal size ${ }^{(28)}$. It reflects the cumulative effect of severe socioeconomic adversities and recurrent infections and produces long-term consequences, including impaired intellectual and school performance and small adult body size ${ }^{(29)}$. In turn, thinness is an indicator of acute malnutrition generally associated with insufficient energy intake, often concurrent with parasitic and other infectious diseases ${ }^{(28)}$. Delayed puberty and reduced muscle strength and bone mass density later in life are some of the significant consequences of thinness during school years ${ }^{(29)}$.

Our findings reveal two faces of undernutrition present in the context of food insecurity: high frequency of thinness and stunting ${ }^{(1)}$. Since the crises of 2008 that raised the prices of food commodities (especially wheat, rice, soya, and maize), an unprecedented increase in the number of hungry people is being observed in Benin $^{(30)}$ and most countries worldwide ${ }^{(31)}$. In addition to this, climate variability and extreme weather like dry seasons or floods have contribute further to acute under nutrition and aggravation of chronic malnutrition, especially in populations depending widely on agriculture ${ }^{(32-33)}$ such as Benin ${ }^{(34)}$. According to the World Food Program of the United Nations, in 2017, food insecurity affected almost $10 \%$ of the Beninese population ${ }^{(11)}$.

The relationship between age and odds of thinness and stunting observed in the present study is in accordance with findings of previous studies ${ }^{(28,35)}$. The end of childhood is an intense linear growth phase, implying increased daily energy (caloric) and nutrient requirements for optimal growth and development. Inability to meet these needs may result in or aggravate existing nutritional deficiencies ${ }^{(36)}$. On the other hand, it is possible that schoolchildren with nutritional deficiencies are retained more often in primary education, influencing the observed results, where school meals are offered. This latter fact may account for the increased odds of stunting among children reporting school meals five versus less days/ week. In 2018, 15\% of primary school schoolchildren in Benin were repeaters ${ }^{(37)}$. The expected age range for primary education in the country would be 6 to 11 years ${ }^{(37)}$, while we found 8 to 17 years, a probable combination of late entry to school and high retention rates.

Our results suggest that higher level of formal maternal education is associated with lower odds of stunting, while formal father education was not associated with thinness or stunting. This result agrees with a study of scholars (6-12 years old) in Nigeria ${ }^{(4)}$. Although both maternal and paternal education is important for reducing childhood ( $<5$ years of age) undernutrition ${ }^{(38)}$, maternal education appears to have deeper impact on children's nutritional and health outcomes owing to improvement of women's welfare, feeding practices and healthcare service use ${ }^{(39)}$. The lack of statistically significant association between household socioeconomic status and nutritional outcomes in this study may result from misclassification errors, due to difficulties of assessing such information via self-administered questionnaire in schoolchildren.

Low dietary diversity was significantly associated with stunting, but not thinness, in this study, a finding in line with the evidence that all diet components are important to prevent chronic undernutrition. Dietary diversity, an indicator of adequate nutrient intake and quality of $\operatorname{diet}^{(22)}$, is vital for optimal health and reduces the risk of nutritional deficiencies ${ }^{(40)}$. On the other hand, short-term episodes of inadequate nutrition, mostly total energy intake, and illness appear more important than diet quality to thinness ${ }^{(41)}$.

Our results support the importance of school meals to reduce food insecurity, as the experience of hunger at school was strongly associated with thinness and stunting. In this regard, reverse causality appears as the most likely explanation for the strong association between school meals five days a week and stunting, instigated by a nutritional intervention started in 2017 in Benin. This year, a National Integrated School Feeding Program began in areas of food insecurity, particularly in schools located in rural areas ${ }^{(42)}$. Since then, receiving meals five days a week became a marker of high stunting prevalence. However, it appears from our study that this program remains of limited reaching, since only $6 \%$ of children reported eating school meals, in contrast with the high prevalence of stunting.

Expanding school-feeding programs is imperative to reach all vulnerable schoolchildren, not only the extremely affected ones, and prevent undernutrition in a country with is one of the highest infant mortality rates in the world ${ }^{(43)}$. Hunger is a major and increasing form of food insecurity ${ }^{(33,44)}$, being this human right a priority target for public health interventions. We must note that health expenditure in Benin is very low (2.5\% of the Gross Domestic Product in 2018) ${ }^{(45)}$, and the numbers of healthcare professionals are scarce $(0.39$ nurses and midwives per 1,000 people; 0.08 physicians per 1,000 people in 2018) ${ }^{(45)}$. Evidence indicate that combining the efforts of educational and healthcare professionals are more effective to adequately monitoring and assist child growth and development on a regular basis, and intervening at early and reversible stages of undernutrition $^{(46)}$. Nursing professionals usually have substantive leading roles in health education and health-promoting school initiatives ${ }^{(47)}$ released by WHO and UNESCO ${ }^{(10)}$.

\section{Study limitations}

Firstly, dietary diversity estimated according to a single 24hour dietary recall may not have reflected the daily and seasonal 
variation of schoolchildren diet. Although self-administered questionnaire enjoys increasing popularity in school-surveys, potential memory biases cannot be ignored. Since the prevalence of the main outcomes were higher than the $10 \%$ used to estimate sample size, we may have been underpowered to detect small or modest differences in nutritional outcomes. Finally, the cross-sectional nature of this study precludes determining the temporality of dietary patterns and nutritional outcomes.

\section{Contributions to nursing, health, or public policy}

The regular assessment and monitoring of the nutritional status of children and adolescents in school surveys are important and feasible strategies. They are essential to subsidy the implementation as well as the assessment of the impact of school health programs directed to promote childhood health and prevent damages in linear growth and cognitive and emotional development of schoolchildren.

\section{CONCLUSIONS}

Undernutrition is a health concern among schoolchildren in Lokossa-Benin, as the prevalence of thinness and especially of stunting is very high. Findings of this study support the importance of food insecurity, particularly food deprivation, to explain thinness and stunting. Low dietary diversity also seems to contribute to chronic malnutrition, while greater maternal education seems to mitigate the problem. Public health interventions, such as far-reaching school meal, in association with poverty reducing programs, are essential to prevent chronic nutritional deficiencies and promote adequate linear growth and full development of schoolchildren of Lokossa.

\section{FUNDING}

Herbert Sagbo and Aline A.B. Costa was a PhD candidate, both supported by CAPES-BR (Finance Code 001). LG, SMB are research fellows of CNPq, Brazil. The funder had no role in the design and collection, analysis or writing of this article.

\section{ACKNOWLEDGMENT}

The authors thank the participants and the staff of the Research on Nutritional and Health Status of Primary Education Students in the City of Lokossa-Benin for their important contributions. The authors also thank the staff of the ELSA Study in Minas Gerais and the Postgraduate Program in Public Health, Faculty of Medicine, Universidade Federal de Minas Gerais for their support.

\section{REFERENCES}

1. Food and Agriculture Organization. The state of food security and nutrition in the world: safeguarding against economic slowdowns and downturns [Internet]. Rome: FAO; 2019[cited 2020 May 05]. Available from: https://www.fao.org/publications/card/en/c/CA5162EN/

2. Dukhi N. Global prevalence of malnutrition: evidence from literature. In: Imran M, Imran A, editors. Malnutrition. London: IntechOpen; 2020. p. 1-17. https://doi.org/10.5772/intechopen.92006

3. United Nations Children's Fund. World Health Organization. World Bank. Joint child malnutrition estimates [Internet]. 2018 [cited 2020 Jan 31]. Available at: http://datatopics.worldbank.org/child-malnutrition/

4. Ndukwu Cl, Egbuonu I, Ulasi TO, Ebenebe JC. Determinants of undernutrition among primary school children residing in slum areas of a Nigerian city. Niger J Clin Pract. 2013;16(2):178-83. https://doi.org/10.4103/11199-3077.110142

5. Getaneh Z, Melku M, Geta M, Melak T, Hunegnaw MT. Prevalence and determinants of stunting and wasting among public primary school children in Gondar town, northwest, Ethiopia. BMC Pediatr. 2019;19(1):207. https://doi.org/10.1186/s12887-019-1572-x

6. United Nations Children's Fund. Multi-sectoral approaches to nutrition: nutrition-specific and nutrition-sensitive interventions to accelerate progress. New York: Unicef; 2014.

7. United Nations Children's Fund. The state of the world's children 2019: children, food and nutrition: growing well in a changing world. New York: Unicef; 2019.

8. Tariq J, Sajjad A, Zakar R, Zakar MZ, Fischer F. Factors associated with undernutrition in children under the age of two years: secondary data analysis based on the Pakistan demographic and health survey 2012-2013. Nutrients. 2018;10(6):676. https://doi.org/10.3390/nu10060676

9. World Health Organization. Nutrition in adolescence: issues and challenges for the health sector: issues in adolescent health and development. Geneva: WHO; 2005.

10. World Health Organization. Making every school a health-promoting school: global standards and indicators for health-promoting schools and systems. Geneva:WHO; 2021.

11. World Food Programme. Benin [Internet]. Cotonou:WFP; 2018[cited 2021 Jul 29]. Available at: https://www.wfp.org/countries/benin

12. Institut National de la Statistique et de l'Analyse Économique (BJ), Enquête Démographique et de Santé au Bénin, $2017-2018$ [Internet]. Cotonou: INSAE; 2019 [cited 2020 Jul 22]. Available at: https://www.insae-bj.org/images/docs/insae-statistiques/enquetes-recensements/ EDS/2017-2018/1.Benin_EDSBV_Rapport_final.pdf

13. Galetti V, Mitchikpè CES, Kujinga $P$, Tossou F, Hounhouigan DJ, Zimmermann MB, et al. Rural Beninese children are at risk of zinc deficiency according to stunting prevalence and plasma zinc concentration but not dietary zinc intakes. J Nutr. 2016;146(1):114-23. https://doi. org/10.3945/jn.115.216606 
14. Commune de Lokossa (BJ). Plan de developpement communal: 2017-2021 [Internet]. Commune de Lokossa; AMBERO; 2017[cited 2019 Oct 10]. Available at: http://www.ancb-benin.org/pdc-sdacmonographies/PDC/Mono/Lokossa/PDC\%20Lokossa.pdf

15. Sagbo H, Ekouevi DK, Ranjandriarison DT, Niangoran S, Bakai TA, Afanvi A, et al. Prevalence and factors associated with overweight and obesity among children from primary schools in urban areas of Lomé, Togo. Public Health Nutr. 2018;21(6):1048-56. https://doi.org/10.1017/ S1368980017003664

16. Ministere de la Sante (BJ). Rapport final: enquête globale sur la santé des élèves au Bénin en 2009 [Internet]. [Porto Novo]: MS; 2009[cited 2017 Mar 20]. Available at: http://www.who.int/ncds/surveillance/gshs/Country_Report_GSHS_BENIN_2009.pdf

17. Instituto Brasileiro de Geografia e Estatística. Pesquisa nacional de saúde do escolar: PeNSE 2015 [Internet]. Brasília, DF: IBGE; 2015[cited 2019 Mar 10]. Available at: https://biblioteca.ibge.gov.br/visualizacao/livros/liv97870.pdf

18. Bloch KV, Szklo M, Kuschnir MCC, Abreu GA, Barufaldi LA, Klein CH, et al. The study of cardiovascular risk in adolescents-ERICA: rationale, design and sample characteristics of a national survey examining cardiovascular risk factor profile in Brazilian adolescents. BMC Public Health. 2015;15:94. https://doi.org/10.1186/s12889-015-1442-x

19. Daboné C, Delisle HF, Receveur O. Poor nutritional status of schoolchildren in urban and peri-urban areas of Ouagadougou (Burkina Faso). Nutr J. 2011;10:34. https://doi.org/10.1186/1475-2891-10-34

20. Conway JM, Ingwersen LA, Vinyard BT, Moshfegh AJ. Effectiveness of the US department of agriculture 5-step multiple-pass method in assessing food intake in obese and nonobese women. Am J Clin Nutr. 2003;77(5):1171-8. https://doi.org/10.1093/ajcn/77.5.1171

21. de Onis M, Onyango AW, Borghi E, Siyam A, Nishida C, Siekmann J. Development of a WHO growth reference for school-aged children and adolescents. Bull World Health Organ. 2007;85(9): 660-7. https://doi.org/10.2471/blt.07.043407

22. Ruel MT. Operationalizing dietary diversity: a review of measurement issues and research priorities. J Nutr. 2003;133(11 suppl 2):3911S-26S. https://doi.org/10.1093/jn/133.11.3911S

23. Kennedy G, Ballard T, Dop M-C, organizers. Guidelines for measuring household and individual dietary diversity. Rome: FAO; 2010.

24. Hanley JA, Negassa A, Edwardes MDB, Forrester JE. Statistical analysis of correlated data using generalized estimating equations: an orientation. Am J Epidemiol. 2003;157(4):364-75. https://doi.org/10.1093/aje/kwf215

25. Saltzman E, Schlossman N, Brown CA, Balan I, Fuss P, Batra P, et al. Nutrition status of primary school students in two rural regions of GuineaBissau. Food Nutr Bull. 2017;38(1) 103-14. https://doi.org/10.1177/0379572116679071

26. Teferi DY, Atomssa GE, Mekonnen TC. Overweight and undernutrition in the cases of school-going adolescents in Wolaita Sodo Town, Southern Ethiopia: cross-sectional study. J Nutr Metab. 2018;2018:8678561. https://doi.org/10.1155/2018/8678561

27. Erismann S, Knoblauch AM, Diagbouga S, Odermatt P, Gerold J, Shrestha A, et al. Prevalence and risk factors of undernutrition among schoolchildren in the Plateau Central and Centre-Ouest regions of Burkina Faso. Infect Dis Poverty. 2017;6(1):17. https://doi.org/10.1186/ s40249-016-0230-x

28. Gorstein J, Sullivan K, Yip R, de Onís M, Trowbridge F, Fajans P, et al. Issues in the assessment of nutritional status using anthropometry. Bull World Health Organ. 1994;72(2):273-83.

29. Best C, Neufingerl N, van Geel L, van den Briel T, Osendarp S. The nutritional status of school-aged children: why should we care?. Food Nutr Bull. 2010;31(3):400-17. https://doi.org/10.1177/156482651003100303

30. République du Bénin. Examen strategique national Faim Zero au Benin à l'horizon 2030 [Internet]. [Porto Novo]: République du Bénin; 2018[cited 2020 Jul 22]. Available at: https://docs.wfp.org/api/documents/WFP\%0000114271/ download/?_ga=2.240609674.868288096.1594520488-257532199.1591488111

31. Golay C. The food crisis and food security: towards a new world food order?. Int Dev Policy.2010;1: 215-32. https://doi.org/10.4000/poldev.145

32. Adjimoti GO, Kwadzo GT-M. Crop diversification and household food security status: evidence from rural Benin. Agric Food Secur. $2018 ; 7: 82$. https://doi.org/10.1186/s40066-018-0233-x.

33. Food and Agriculture Organization of the United Nations. The state of food security and nutrition in the world 2018: building climate resilience for food security and nutrition. Rome: FAO; 2018.

34. World Food Programme. Benin Country Brief [Internet]. [Porto Novo]:WFP; 2020[cited 2020 Jul 22]. Available at: https://reliefweb.int/report/ benin/wfp-benin-country-brief-may-2020

35. Mustaq MU, Gull S, Khurshid U, Shahid U, Shad MA, Siddiqui AM. Prevalence and socio-demographic correlates of stunting and thinness among Pakistani primary school children. BMC Public Health. 2011;11:790. https://doi.org/10.1186/1471-2458-11-790

36. Akombi BJ, Agho KE, Hall JJ, Wali N, Renzaho AMN, Merom D. Stunting, wasting and underweight in sub-Saharan Africa: a systematic review. Int J Environ Res Public Health. 2017;14(8):863. https://doi.org/10.3390/ijerph14080863

37. United Nations Educational. Benin [Website]. 2020[cited 2020 Jun 26]. Available at: http://uis.unesco.org/en/country/bj\#slideoutmenu

38. Vollmer S, Bommer C, Krishna A, Harttgen K, Subramanian SV. The association of parental education with childhood undernutrition in low- and middle-income countries: comparing the role of paternal and maternal education. Int J Epidemiol. 2017;46(1):312-23. https://doi. org/10.1093/ije/dyw133

39. Makoka D. The impact of maternal education on child nutrition: evidence from Malawi, Tanzania, and Zimbabwe. Calverton: ICF International; 2013. (DHS working papers, № 84). 
40. Wells JC, Sawaya AL, Wibaek R, Mwangome M, Poullas MS, Yaynik CS, et al. The double burden of malnutrition: aetiological pathways and consequences for health. Lancet. 2020;395(10217):75-88. https://doi.org/10.1016/S0140-6736(19)32472-9

41. Popkin BM, Corvalan C, Grummer-Strawn LM. Dynamics of the double burden of malnutrition and the changing nutrition reality. Lancet. 2020;395(10217):65-74. https://doi.org10.1016/S0140-6736(19)32497-3

42. Jomaa LH, McDonnell E, Probart C. School feeding programs in developing countries: impacts on children's health and educational outcomes. Nutr Rev. 2011;69(2):83-98. https://doi.org/10.1111/j.1753-4887.2010.00369.x

43. The Borgen Project. Improving health and healthcare in Benin [Internet]. [place unknown]: The Borgen Project; 2020 Sept 15 [cited 2021 Jul 28]. Available at: https://borgenproject.org/healthcare-in-benin/

44. Niclasen B, Petzold M, Schnohr CW. Adverse health effects of experiencing food insecurity among Greenlandic school children. Int J Circumpolar Health. 2013;72. https://doi.org/10.3402/ijch.v72i0.20849. https://doi.org/10.3402/ijch.v72i0.208

45. World Bank. Indicators [Internet]. [place unknown]: The Bank; c2021 [cited 2021 Jul 28]. Available at: https://data.worldbank.org/indicator/

46. Falbo BCP, Andrade RD, Furtado MCC, Mello DF. Estímulo ao desenvolvimento infantil: produção do conhecimento em enfermagem. Rev Bras Enferm. 2012;65(1):148-54. https://doi.org/10.1590/S0034-71672012000100022

47. Whitehead D. The health-promoting school: what role for nursing?. J Clin Nurs. 2006;15(3):264-71. https://doi.org/10.1111/j.1365-2702.2006.01294.x 